\title{
Estrategias básicas de marketing para microempresarios.
}

\section{Basic marketing strategies for microenterprises.}

Ing. Alberto Efraín Prado Chinga, MBA ${ }^{1}$, Ing. Kleber Santos Guerra Herrera, MBA. ${ }^{2}$, Ing. Pamela de Lourdes Ortega Ojeda, $\mathrm{MBA}^{3}$, \& Ing. Carlos Omar Gutiérrez Ruano, MSc. $^{4}$

DOI: https://doi.org/10.33262/visionariodigital.v3i2.398

\section{Resumen.}

El marketing es importante para mejorar las oportunidades futuras mediante la implementación de técnicas que permitan a los microempresarios tener un alto desempeño. Razón por la cual, la presente investigación se desarrolla un manual de técnicas básicas de marketing para los microempresarios, los cuales se ha visto afectada por la falta de conocimientos al no contar estrategias que les permita mejorar desempeño comercial y alinear sus estrategias al logro de sus objetivos que les permita ser más competitivo en su actual entorno. Teniendo en cuenta la problemática planteada que se ha presentado por la carencia de conocimientos de técnicas de marketing alineado a su realidad, se asiste al diseño de campo de carácter no experimental y con un enfoque cualitativo para recabar datos primarios directamente donde se originan los hechos. Por tales motivos, se aplicó la encuesta escrita a los microempresarios, seleccionados a través del muestreo no probabilístico intencional debido a que la población es finita. De la misma forma, se aplicó el análisis de las necesidades del microempresario como herramienta para priorizar los resultados obtenidos de los cuestionarios aplicados para evaluar la perspectiva de los comerciantes, denotando la necesidad de adquirir conocimientos de marketing. Por tanto, se propuso la propuesta de un manual de técnicas básicas de marketing alineados a la compañía a los microempresarios, que permitan su desarrollo.

Palabras claves: Técnicas de marketing, objetivos, indicadores, metas, estrategias.

\footnotetext{
${ }^{1}$ Universidad Técnica Luis Vargas Torres de Esmeraldas Sede La Concordia, alberto.prado@utelvt.edu.ec

${ }^{2}$ Servicio Nacional de Gestión de Riesgos y Emergencias Delegación Provincial Santo Domingo de los Tsáchilas, kleber.guerra@gestionderiesgos.gob.ec

${ }^{3}$ Universidad Técnica Luis Vargas Torres de Esmeraldas Sede La Concordia, pameort@gmail.com

${ }^{4}$ Universidad Técnica Luis Vargas Torres de Esmeraldas Sede La Concordia, gcarlosguter@hotmail.com
} 


\begin{abstract}
.
Marketing is important to improve future opportunities through the implementation of techniques that allow microentrepreneurs to have a high performance. Reason for which, the present research develops a manual of basic marketing techniques for microentrepreneurs, which has been affected by the lack of knowledge by not counting strategies that allow them to improve business performance and align their strategies to achieve their goals. objectives that allow them to be more competitive in their current environment. Taking into account the problem posed that has been presented by the lack of knowledge of marketing techniques aligned with their reality, we assist in the design of a non-experimental field and with a qualitative approach to collect primary data directly where the facts originate. For these reasons, the written survey was applied to microentrepreneurs, selected through intentional non-probabilistic sampling because the population is finite. In the same way, the needs analysis of the microentrepreneur was applied as a tool to prioritize the results obtained from the questionnaires applied to evaluate the perspective of the merchants, denoting the need to acquire marketing knowledge. Therefore, the proposal of a manual of basic marketing techniques aligned with the company to microentrepreneurs, which allow their development, was proposed.
\end{abstract}

Keywords: Marketing techniques, objectives, indicators, goals, strategies.

\title{
Introducción.
}

Las técnicas básicas de marketing son estrategias empeladas para lograr varias metas en las empresas estas pueden ser que los clientes mejoren la visión sobre la empresa, incremento de las ventas, promoción constante productos y servicios. Es imprescindible conocer estos objetivos, así las personas puedan entender el motivo por el cual es indispensable el uso de estrategias de marketing. De acuerdo con la visión del "Padre del Marketing”, el marketing es una función empresarial que busca identificar necesidades y deseos no satisfechos. Agrega que el área define y también muda la magnitud y su potencial de rentabilidad (Kotler \& Armstrong, 2013). Otro punto relacionado al marketing acorde el autor es la especificación de mercados objetivos El marketing requiere razonar las decisiones sobre los productos y servicios. En general, es una estrategia que complementa los procesos de ventas y el trato entre la empresa y los clientes. Se concluyó, que, es importante que todo microempresario debe tener conocimiento de estrategias básicas de marketing no solo para mejorar las ventas, sino para captar las necesidades del consumidor para el desarrollo empresarial, asimismo cada microempresario tendrá en cuenta que metas quiere alcanzarla empresa y estará comprometido con seguir ese objetivo, exponiéndose así, la relevancia del presente tema de investigación (Peñaloza, 2005). (Kotler \& Armstrong, 2013)

El marketing es importante para tratar las oportunidades de crecimiento comercial mediante la elaboración estrategias que permitan tener un alto alcance en ventas. Razón por la cual, el uso de técnicas de marketing es necesario al ser la forma más certera de 
impactar en el mercado teniendo en cuenta el producto, el precio, el empaque y el lugar. De la misma forma, permite controlar los objetivos fijados (Gallardo , 2013).

Actualmente, las PYMES a nivel mundial fracasan porque no consiguen acaparar la suficiente cantidad de clientes, o porque se dirigen a equivocados tipos de clientes que solo compran por precios, un contexto altamente competitivo y cambiante en los nuevos mercados, en donde los modelos tradicionales de negocios son afectados por un mundo donde la innovación es parte fundamental del éxito, implementar estrategias de marketing que les permitan alcanzar los resultados deseados con los recursos que dispone la entidad, además, se toma en consideración que sin estrategias las pymes no tienen cabida a largo plazo y se ven afectada por diversos factores externos e internos como la crisis económica, variaciones en el mercado, cambios en las actividades económicas o la creación de nuevos productos o servicios. Varios microempresarios mantienen conceptos distorsionados sobre la implementación de estratégicas de marketing y rechazan la idea de incorporarlas en sus organizaciones y otros los consideran sin ningún beneficio, sin tomar en cuenta el aporte significativo en sus ventas. (Farias, 2014)

En el Ecuador, las pymes dedicadas a la venta al por mayor y menor, realizan sus actividades de manera empírica al no contar con conocimientos de estrategias de marketing que les permita medir su nivel ventas y el cumplimiento de metas a corto, mediano y largo 3 plazo. Puesto que, la mayoría de los microempresarios no cuentan con la capacidad de implementar nuevas estrategias que les permita crear ventajas competitivas con planeación. Además, tales organizaciones se han visto afectadas por los cambios socios económicos, políticos, tecnológicos y legales en las últimas décadas que han conllevado a la búsqueda de nuevas estrategias que contribuyan al giro del negocio, sin embargo, por el desconocimiento de estrategias de marketing, se ha evidenciado la perdida de espacios competitivos, de recursos y en el peor de los casos el cese de actividades (Alcaide, y otros, 2013).

Partiendo de lo antes mencionado, la presente investigación se ejecuta en los 40 microempresarios, tomando en consideración que su actividad económica actual es la venta al por menor de productos de consumo masivo, la cual se ha visto afectada por cambios externos e interno al no contar con los suficientes conocimientos de estratégicas de marketing que les permita medir su nivel de competitividad e incrementar sus ventas (Andrade, 2016). Además, teniendo en consideración una visión largo plazo es la respuesta que le permite a los microempresarios definir de forma efectiva las estrategias necesarias dependiendo de su actividad. Se presenta a continuación en la figura uno las causas y efectos más palpables a los que actualmente se presentan en los microempresarios, por la falta de conocimientos de estrategias de marketing.

\section{Causa y efecto del problema.}

El plan de marketing en la pyme es una de navegación clara y concisa en la que resulta fácil situarse, descubrir todos los elementos que nos rodea, intuir todas las amenazas y oportunidades y, en las líneas generales, te enseña que la innovación es el norte de toda 
acción. (Alcaide, et.al, 2013). Por tanto, la creación de manual de técnicas básicas de marketing para los 40 microempresarios, se ejecutará por la falta de conocimientos que 5 contribuyan a mejorar sus ventas y por la necesidad de crear una ventaja competitiva en el mercado actual. Por tanto, las técnicas de marketing básicas es una herramienta que permite servir como guía práctica creando nuevas estrategias de mercadeo con el propósito que los microempresarios les permitan aumentar las ventas (Andrade , 2016).

La investigación se desarrollará mediante el diseño de acción como estrategia para reconocer al problema, dificultad o conflicto antes mencionado, dicho diseño de enfoque cualitativo se ejecuta en el estudio de la realidad, en el proceso de interpretación y situaciones observadas con el objetivo de generar datos para su correcta descripción y análisis.

Las estrategias básicas de marketing causan su mayor impacto cuando los microempresarios logran comprender las estrategias que deben implementar en el mercado. Los propietarios deben establecer objetivos a largo, corto y mediano plazo en ventas para sí lograr un crecimiento constante de la empresa. Por tanto, es importantes para los microempresarios, estratégicas que contribuyan a la eficiencia y eficacia de su desempeño. Además, tal propuesta podrá ser incorporada para cualesquiera pymes que tenga la misma problemática. (Rivera, 2015)

En la presente investigación se establece los objetivos con el propósito de evaluar la perspectiva empresarial de los microempresarios para comprender las estrategias que se debe implementar en el mercado, con la finalidad de diseñar un manual de técnicas básicas de marketing que ayuden al mejoramiento de sus ventas. De la misma forma, con apoyo de los conocimientos de marketing adquiridos al largo de nuestra carrera logramos identificar cuáles son las técnicas básicas de marketing que los microempresarios deberían conocer para aumentar sus ventar y mejorar su nivel de vida.

\section{Métodos y materiales.}

Conforme a los conocidos antecedentes presentados sobre la problemática del estudio y el lugar donde ocurren los eventos para la obtención de datos primarios y secundarios, se escoge un diseño de campo de carácter no experimentar con enfoque mixto, Por consiguiente, en la presente investigación se utiliza el muestro no probabilístico intencional donde el estudio de los elementos es escogido bajo las razones del investigador, tomando en cuenta que la población en su totalidad es finita por estar compuesto por 40 microempresarios del sector de La Concordia

\section{Análisis de resultados.}

Por consiguiente, seleccionamos las técnicas e instrumentos para conseguir datos primarios en el lugar donde se presenta la problemática de estudio de los 40 microempresarios, y se aplicó una encuesta escrita con el motivo de mejorar y justificar la investigación de campo de carácter no experimental. 
Gráfico. 1 años lleva siendo comerciante.
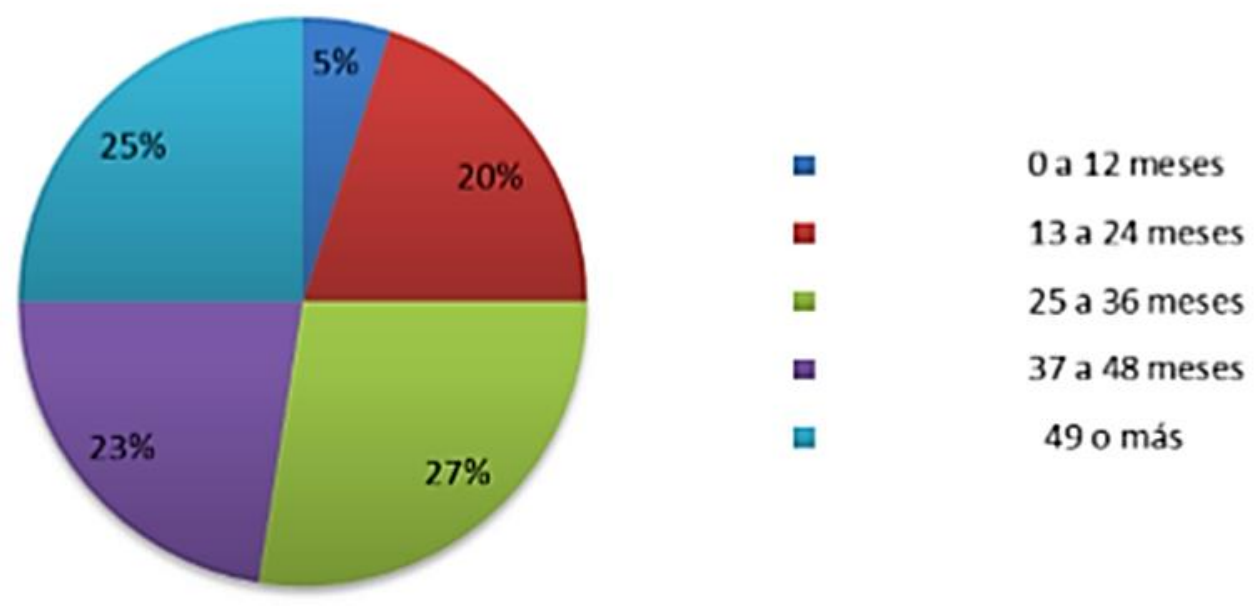

Del total encuestado un $27 \%$ de los microempresarios del centro de duran tienen entre 25 a 36 meses de experiencia como comerciantes, con 49 meses o más de experiencia cuenta el $25 \%$ de nuestra muestra, el $23 \%$ son de 37 a 48 meses de experiencia por otra parte el $5 \%$ cuenta de 0 a 12 meses de experiencia, estos nos demuestran varios años desarrollando en este tipo de actividades.

Gráfico. 2 Nivel de estudio.

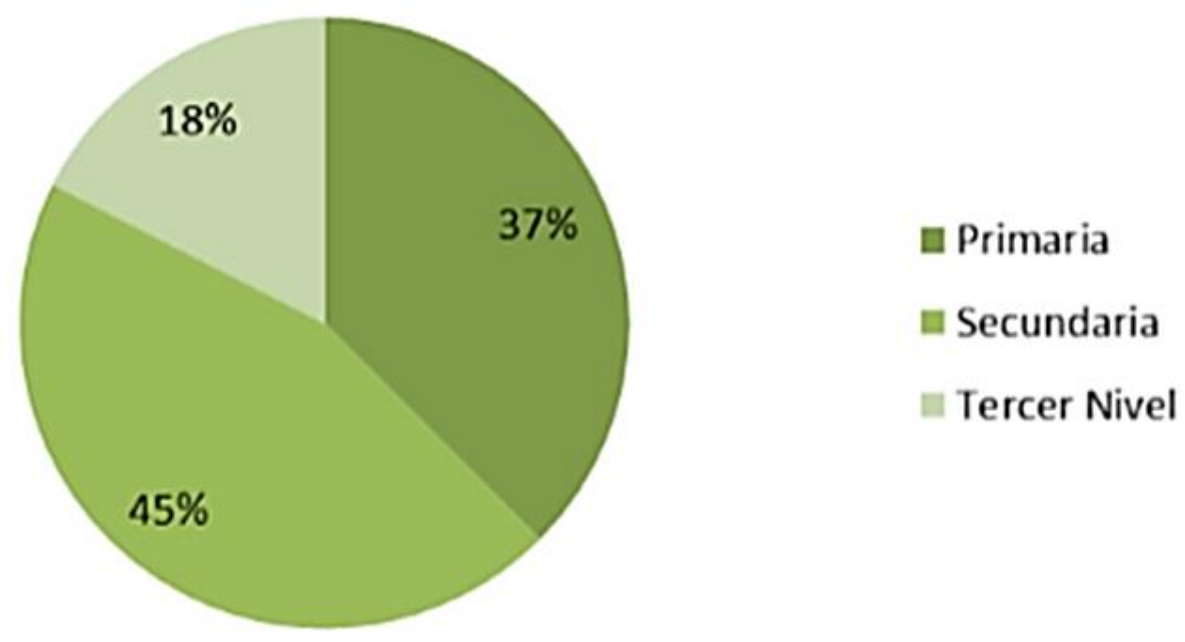

El $45 \%$ de los microempresarios encuestados, cuenta con un nivel de estudio de secundaria, el $37 \%$ los comerciantes constan con un nivel de estudio de primaria por último el $17.5 \%$ han culminado el tercer nivel de estudio. 
Gráfico. 3 conocimientos de marketing.

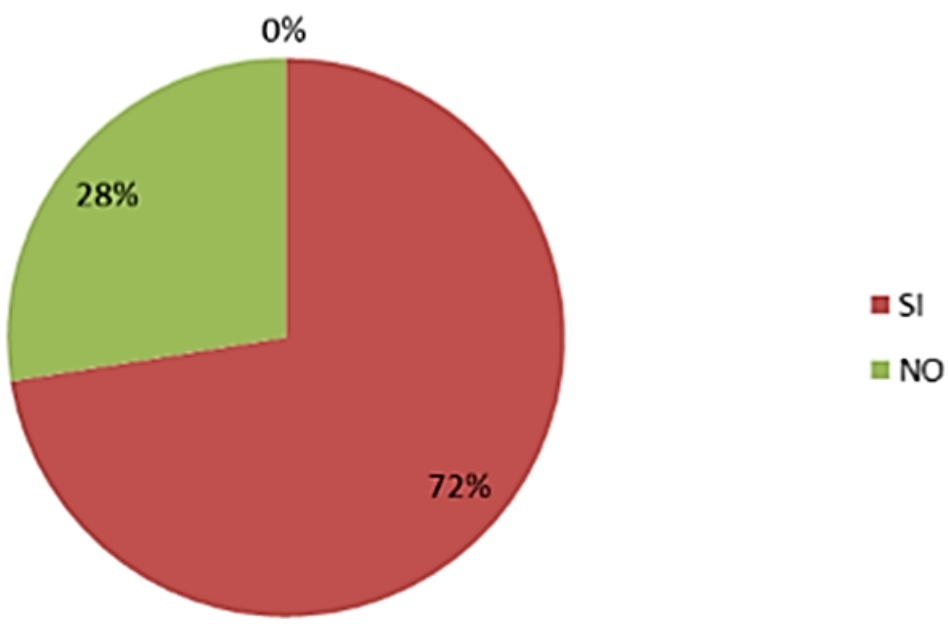

De acuerdo a esta pregunta realizada a los comerciantes el $72.5 \%$ de los microempresarios encuestados creen que mejorarían sus ventas teniendo más conocimientos acerca de marketing y el $27.5 \%$ considera que no.

Gráfico. 4 Métodos probados para el establecimiento y control de las estrategias.

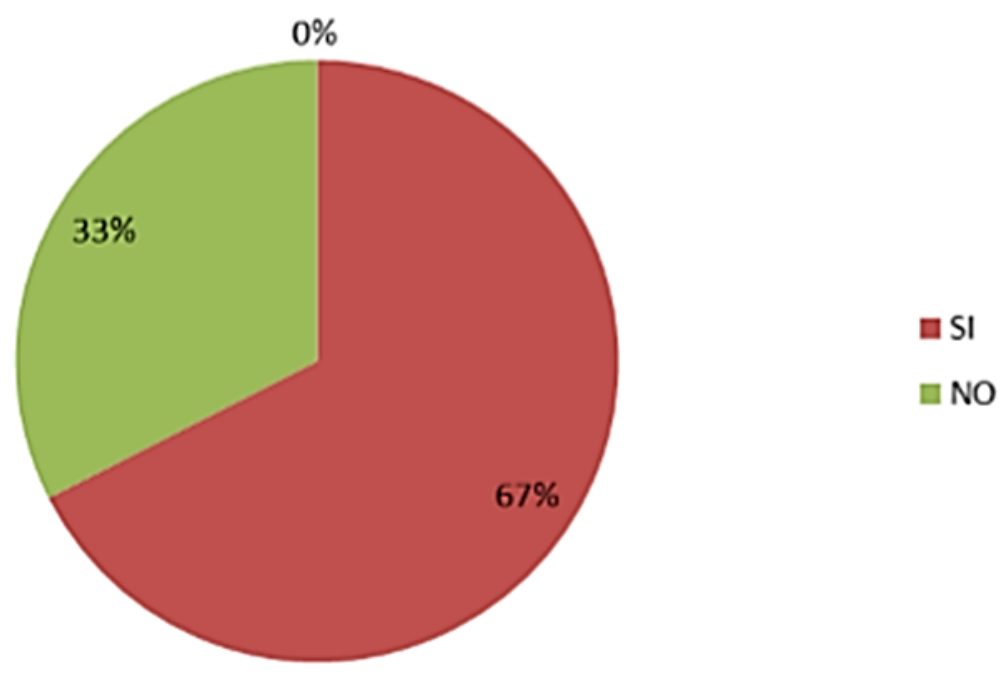

El $67 \%$ de los microempresarios encuestado, están dispuesto a querer aprender sobre las técnicas de marketing y el $33 \%$ piensan que no. 
Gráfico. 5. Implementar las técnicas de marketing.

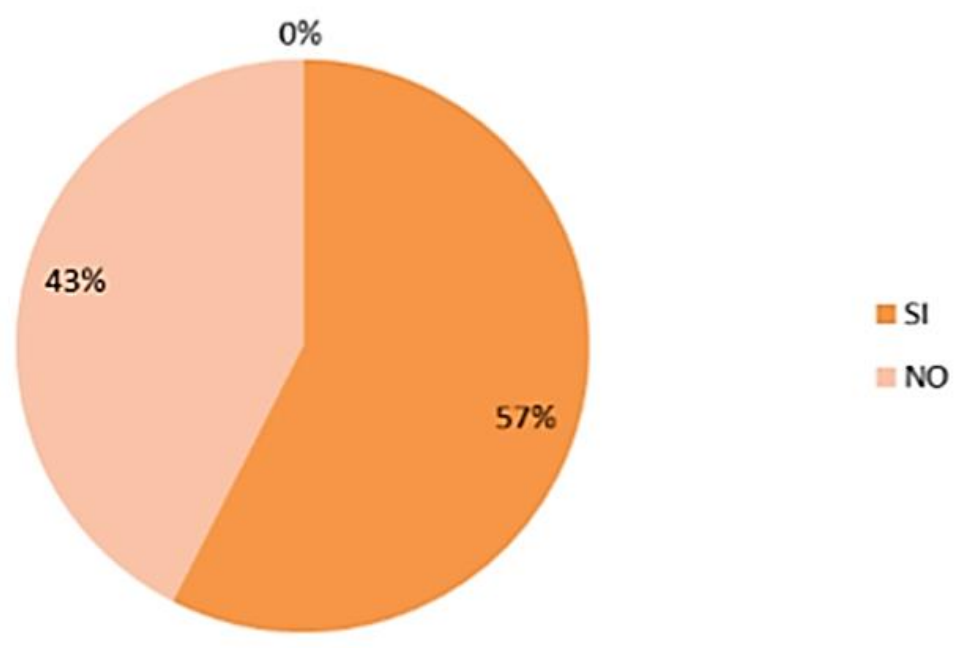

El 57\% de los microempresarios encuestado están de acuerdo en implementar técnicas de marketing y el $43 \%$ de los comerciantes no creen que es no necesario establecer técnicas de marketing.

Gráfico. 6 Manual de técnicas básicas de marketing.

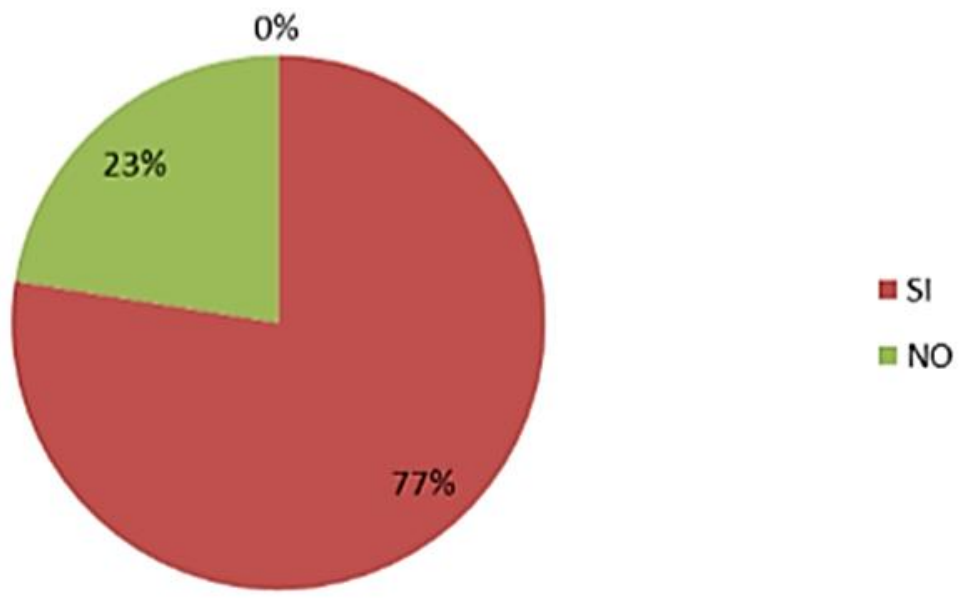

El 77\% de los microempresarios encuestados están dispuestos a leer el manual de técnicas básicas de marketing y el $23 \%$ no están preparados. 
Gráfico. 7 Incremento de ventas mediante el manual de técnicas básicas de marketing.

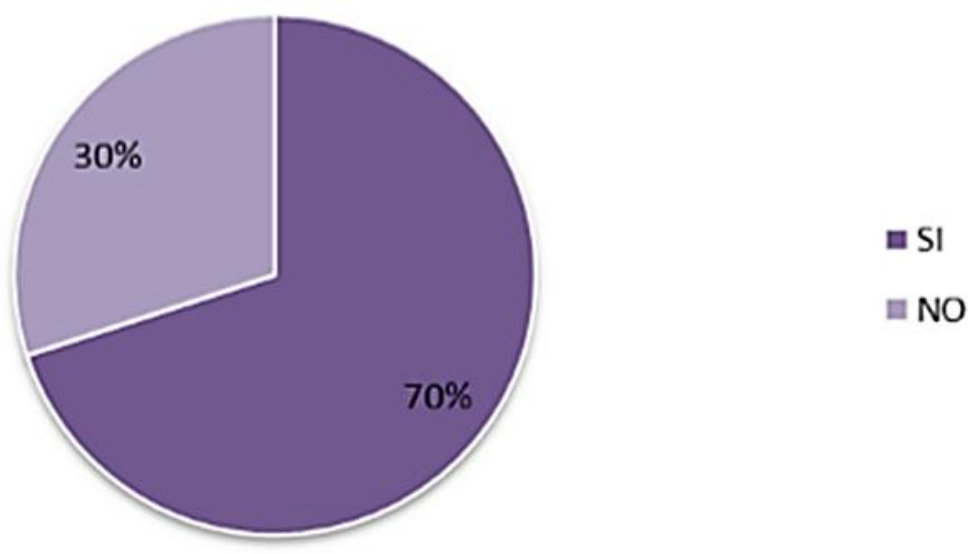

El 70\% de los microempresarios encuestados, consideran que el manual de técnicas básicas de marketing ayudara aumentar sus ventar y tener una mejor rentabilidad y el $30 \%$ considera que un manual de técnicas básicas de marketing no ayudaría en su negocio.

\section{Análisis general.}

Mediante los resultados obtenidos a través de la encuesta escrita se pudo conocer que más

del $50 \%$ de la muestra tienen más de 3 años de experiencia en el sector comerciante, el $18 \%$ de la misma tiene educación de tercer nivel, el $72 \%$ de los encuestados consideran que sus ventas mejorarían si tuvieran conocimientos de marketing y están dispuestos adquirir dichos conocimientos. La mayoría de los comerciantes están dispuestos a leer el manual de técnicas de marketing para el desarrollo de sus ventas. Se ha determinado que la implementación del manual seria de esencial ayuda hacia los comerciantes ya que se podrán establecer metas de ventas a corto, mediano o largo plazo en el mercado mediante estrategias que determinen las necesidades del consumidor y mejorar en el desarrollo empresarial. Dicha estrategia será una ayuda con una gran aceptación por el microempresario ya que ellos necesito de constante innovación para poder mejorar su imagen, en un mercado, que cada día es más difícil competir.

\section{Técnicas básicas de marketing para microempresarios.}

\section{Que es marketing.}

Es un proceso que estudia el comportamiento de los mercados y los consumidores con la finalidad de captar clientes mediante la satisfacción de deseos y necesidades. El área de marketing es vital no solo para el éxito de una empresa, sino también para su existencia. Sin ella, la empresa no podría sobrevivir. (Kotler \& Armstrong, 2013). 


\section{Marketing directo.}

Consiste en contacto personalizado con los clientes con el fin de mantener la relación, cliente propietario de esta forma aumentar la fidelidad.

\section{Marketing relacional.}

Se identifica a los clientes más representativos y se busca obtener una relación de amistad

\section{Marketing virtual.}

Se utilizan el mundo virtual para buscar llegar a más posibles consumidores.

\section{Las estrategias de marketing se basan en las 4P las cuales son:}

Producto $\cdot$ Cuando se venda un producto es necesario que tengo algo que lo diferencia a la competencia, para que los consumidores lo prefieran.

Precio $\bullet$ Es necesario investigar los precios que se manejan en el mercado

Plaza -Se debe establecer un punto de venta estratégico donde nuestros posibles consumidores puedan encontrar el producto.

Promoción • Es la forma principal de mejorar las ventas

\section{Estrategias de marketing para pymes (pequeñas y medianas empresas)}

En la actualidad, se ha podido observar que las pequeñas y medianas empresas se ven afectadas ya que se enfrentan a grandes competencias, por lo cual se muestra varios ejemplos de cómo una Pyme se puede afrontar mediante las estrategias de mercadotecnia adecuadas.

Estrategia. - Se refiere al conjunto de acciones que ayudan a implementar en la toma de decisiones con el fin de lograr el objetivo propuesto de un negocio.

\section{Segmentación del mercado adecuada.}

Es necesario conocer y saber las necesidades y preferencia de su mercado meta para lograr atenderlos de la mejor manera.

\section{Encontrar tu producto estrella.}

Ofrecer un solo tipo de producto de excelente calidad y así los consumidores logren tener una percepción elevada del valor del producto o servicio.

\section{Tener una diferenciación.}

Cuando existe demasiada competencia para una pyme, es importante ofrecer algo diferente y novedoso que los distingan de los demás competidores 
Usar testimonios.

El uso de testimonio de clientes importantes o satisfechos es una estrategia que ayuda a competir de una manera equitativa con cualquier empresa.

\section{Tener presencia en línea.}

Una de las formas efectivas para promocionar su producto o servicio es mediante la creación de una página web con un diseño llamativo y de valor con imágenes de calidad

\section{Realizar campañas Email.}

Son herramientas que ayudan a intercalar conversaciones y así obtener nuevos clientes para mantenerlos interesados en el producto o servicio.

\section{Aprovechar las redes sociales.}

Nos permite captar más clientes, promocionar la marca generando más confianza, obteniendo gran importancia en el mercado meta y un excelente posicionamiento de marca.

\section{Crear alianzas estratégicas.}

Averiguar otros emprendedores para el analices de intercambio que permita dar un giro a la empresa.

\section{Brindar atención personalizada.}

Consiste en asesorar al cliente en la compra del producto o servicio que realmente necesite o diseñar un producto con sus preferencias.

\section{Compensar al cliente.}

Debido a que pymes tienen una cantidad manejable de clientes pueden aprovechar para compensarlos con obsequios pequeños pero atractivos para mantener una buena comunicación y lograr su fidelización.

\section{Recuerde.}

Las técnicas de marketing pueden influenciar positivamente en el desarrollo de tu empresa, pero es necesario ser constante en la aplicación de las mismas.

\section{Conclusiones.}

- El manual de marketing es una herramienta que puede corregir los errores que cometen los microempresarios y tomar las decisiones correctas para la promoción y comunicación de los servicios y productos que ofreces sus negocios.

- Es importante que todos los propietarios de microempresas promuevan su sentido de pertenencia, compromiso, respeto mutuo y diálogo abierto, lealtad y ética. Los 
valores y las metas se establecen para garantizar que los productos y serbios que ofrecen se promocionen de manera responsable y completa.

- Los propietarios de microempresas deben implementar estrategias de descuento para estrategias de pago, estrategias creativas de fijación de precios y estrategias para crear servicios complementarios y estrategias de comunicación. Esto se debe a que los clientes están motivados para consumir y comprar los servicios y/o productos.

- El manual de técnica básicas de marketing se puede encontrar donde los planes promocionales y comunicación de cada servicio o productos se basan en el análisis empresarial de los negocios.

\section{Referencias Bibliográficas.}

Alcaide, J., Bernués, S., Díaz, E., Espinosa, R., Muñiz, R., \& Smith, C. (2013). Marketing y PYMES (primera ed.). Madrid: Copyright. Obtenido de http://www.marketingypymesebook.com/wpcontent/uploads/2013/04/MARKETING-Y-PYMES.pdf

Andrade, D. (2016). Estrategias de marketing digital. Rev. esc.adm.neg., 1(80), 59-72. Obtenido de http://www.scielo.org.co/pdf/ean/n80/n80a05.pdf

Farias, P. (2014). Estrategias de marketing utilizadas por las empresas chilenas para incrementar el valor de los clientes. Cuadernos de Administración, 30(51), 7. Obtenido de http://www.scielo.org.co/pdf/cuadm/v30n51/v30n51a02.pdf

Gallardo, L. (2013). El significado de las variables del marketing-mix para los públicos objetivo. Razón y Palabra, 18(83). Obtenido de https://www.redalyc.org/pdf/1995/199527531043.pdf

Kotler, P., \& Armstrong, G. (2013). Fundamentos de marketing. México: Printed in Mexico.

Obtenido

de https://www.academia.edu/32749954/Fundamentos_de_marketing_11ed_Kotler _Libro_

Peñaloza, M. (2005). El Mix de Marketing: Una herramienta para servir al cliente. Actualidad Contable Faces, 8(10), 71-81. Obtenido de https://www.redalyc.org/pdf/257/25701007.pdf

Rivera, M. (18 de diciembre de 2015). La evolución de las estrategias de marketing en el entorno digital: implicaciones jurídicas. Tesis doctoral Universidad Carlos III de Madrid. Getafe, España. Obtenido de https://earchivo.uc3m.es/bitstream/handle/10016/22498/rosario_rivera_tesis.pdf

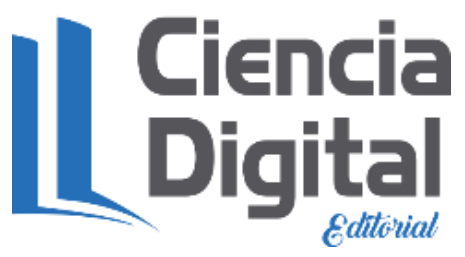




\section{Para citar el artículo indexado.}

Prado A., Guerra K., Ortega P., \& Gutiérrez C. (2019). Estrategias básicas de marketing para microempresarios. Revista electrónica Visionario Digital 3(2), 114-125. Recuperado desde:

http://www.cienciadigital.org/revistascienciadigital/index.php/VisionarioDigital/article/view/3 $\underline{98 / 891}$

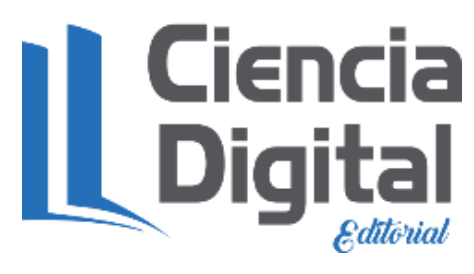

El artículo que se publica es de exclusiva responsabilidad de los autores y no necesariamente reflejan el pensamiento de la Revista Ciencia Digital.

El articulo queda en propiedad de la revista y, por tanto, su publicación parcial y/o total en otro medio tiene que ser autorizado por el director de la Revista Ciencia Digital. 